\title{
Ossicular reconstruction of incudo-stapedial joint by glass ionomer-a study of 24 cases
}

Meenesh Juvekar ${ }^{1,2^{*}}$ (D) and Baisali Sarkar ${ }^{3}$ (D)

\begin{abstract}
Background: Chronic otitis media is one of the major health issues worldwide resulting in partial or complete loss of conductive hearing mechanism including the tympanic membrane and ossicular assembly. The aim of the study is to assess hearing improvement after reconstructing the incudo-stapedial joint with glass ionomer cement. Here, a prospective observational study was done in a tertiary care hospital. Patients of chronic mucosal otitis media were operated, and intraoperatively incudo-stapedial joint discontinuity was restored using glass ionomer cement. Postoperative follow-up was done at 1-month, 3-month, 6-month, and 1-year intervals, and hearing was evaluated by doing pure tone audiometry with air conduction at $0.5,1,2,3,4,6$, and $8 \mathrm{kHz}$ and bone conduction at $0.5,1,2,3$, and $4 \mathrm{kHz}$. Tabulation was done by calculating the air-bone gap in each patient.
\end{abstract}

Results: In our study, the patients showed statistically significant improvement in air conduction thresholds and near closure of air-bone gap post-operatively. The mean AC threshold is $15 \mathrm{~dB}$ with $91.67 \%$ patients having closure of $A B G<20 \mathrm{~dB}$ at the end of the 3rd month. Hearing was also evaluated at 6 -month and 1 -year intervals, which showed good improvement in hearing levels.

Conclusions: Glass ionomer is a simple, physiological, and cost-effective method of tympano-ossicular reconstruction with certain significant post-operative hearing improvement.

Keywords: Hearing loss, Audiometry, Glass ionomer cement, Incudo-stapedial joint, Ossiculoplasty

\section{Background}

Chronic otitis media is one of the major health issues worldwide resulting in partial or complete loss of conductive hearing mechanism including the tympanic membrane and ossicular assembly. Patients often present in the advanced stages of the disease with symptoms of hearing loss that may range up to $60 \mathrm{~dB}$ [1]. Chronic otitis media may be with or without frank cholesteatoma with ossicular erosion. The long process of the incus is the most common site of ossicular erosion with or without an intact stapes suprastructure [2,3]. The main aim of tympano-ossicular reconstruction is to maintain the

\footnotetext{
*Correspondence: meeneshj@gmail.com

2 Juvekars Nursing Home, 17, 18 Shivsagar Society, Shivpuri, Off Sion Trombay Road, Chembur Naka, Chembur, Mumbai, Maharashtra 400071, India

Full list of author information is available at the end of the article
}

normal ossicular assembly between the tympanic membrane and stapes footplate [4]. The aim of this study is to assess hearing improvement after reconstructing the incudo-stapedial joint with glass ionomer cement. Glass ionomer cement comprises inorganic glass particles surrounded by an insoluble hydrogel matrix. It is biocompatible, biostable, moldable, cost-effective, and universally available [5-9]. It is used in dentistry and its usage in ossiculoplasty has been reported [10-12]. This study is necessary to access the post-operative hearing improvement and how much reliable the procedure is.

\section{Methods}

This is a prospective observational study conducted on patients attending the otorhinolaryngology department of tertiary care hospital in Mumbai, West India, from February 2016 to February 2018. 
Inclusion criteria included patients aged between 15 and 55 years, attending ENT OPD, with conductive hearing loss. Microscopic examination showed a mucosal type of chronic otitis media. A high-resolution CT scan of the temporal bone showed a suspicious long process of incus necrosis without any evidence of any obvious cholesteatoma. Pure tone audiometry correlated with ossicular discontinuity with average AC threshold $>40$ to $50 \mathrm{~dB}$ hearing loss. Intraoperatively, the findings were confirmed. Patients having incudo-stapedial discontinuity with erosion less than $1 / 3 \mathrm{rd}$ of the long process of the incus were selected for this procedure (Fig. 1).

Exclusion criteria included patients having otosclerosis, cholesteatoma, tympanosclerosis, erosion of more than $1 / 3$ rd of the long process of the incus, middle ear effusion, and immunocompromised conditions.

Selected patients were admitted 1 day before surgery and detailed history, clinical examination, and microscopic examination were done. A pre-operative CT scan of the temporal bone and pure tone audiometry were evaluated and written informed consent taken.

Under general anesthesia, tympanoplasty or mastoidectomy was done via a post-auricular approach. The tympanomeatal flap was elevated and the postero-superior bony overhang was removed to expose the ossicular chain. The middle ear was inspected for ossicular mobility and other diseases. A cortical mastoidectomy was done in certain indicated cases. Care was taken not to expose more than $2 / 3$ rd of the incus, which could later result in slow postero-superior retraction of the tympanic membrane. In patients where more than $2 / 3 \mathrm{rd}$ of the incus was exposed, a cartilage was kept in the postero-superior quadrant to prevent any retraction pocket in the future. Those patients who were diagnosed to have incudo-stapedial discontinuity intraoperatively with less than $1 / 3$ rd of the long process of incus necrosis were

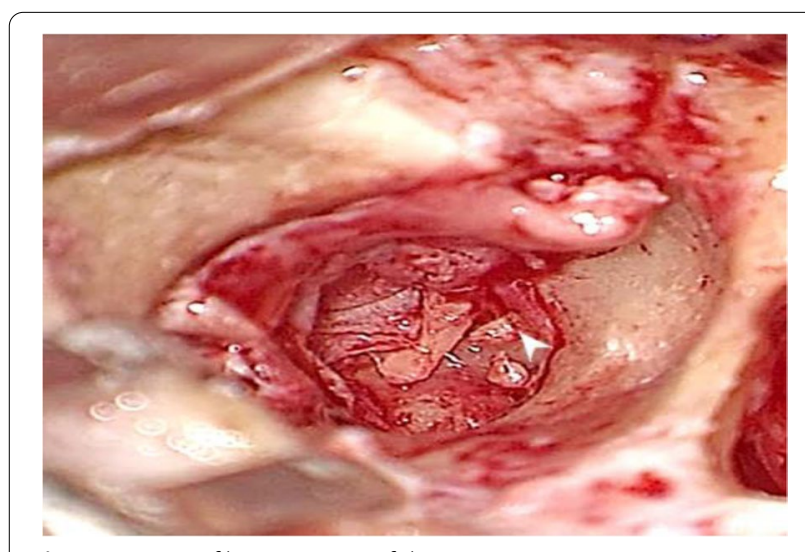

Fig. 1 Erosion of long process of the incus included in the study (Fig. 1). The irregular/necrosed end of incus was freshened, nibbled, and cut with a House Dieter malleus nipper. This area was cleared of blood and good hemostasis achieved. Gelfoam was placed in the middle ear and below the incudo-stapedial joint to prevent any spillage of glass ionomer on stapes footplate, stapes suprastructure, and middle ear (Fig. 2).

Glass ionomer is made up of sterile powder and liquid. In our study, slow binding glass ionomer was used which gives some time to align the incudo-stapedial joint. Both were mixed on a sterile glass slide for $10 \mathrm{~s}$ and used within 2 to $3 \mathrm{~min}$. The mixture was placed between the incus and stapes by using a thin pick. A time period of 7 to $10 \mathrm{~min}$ is required for the fixation of the mixture on the joint. Warm air may be used near the ear for quick setting of the glass ionomer. The ossicular mobility was checked (Fig. 3). Protective gelfoam under the IS joint

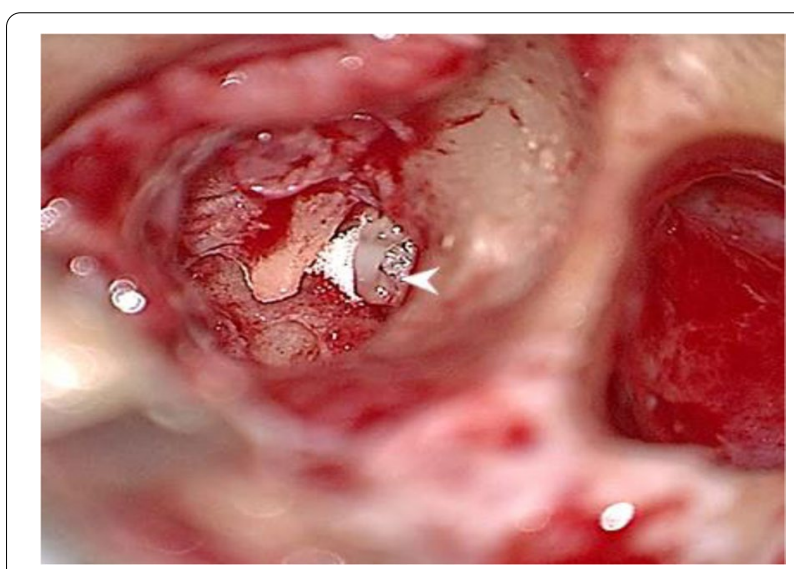

Fig. 2 Gelfoam placed below the incudo-stapedial joint to prevent spillage of the glass ionomer

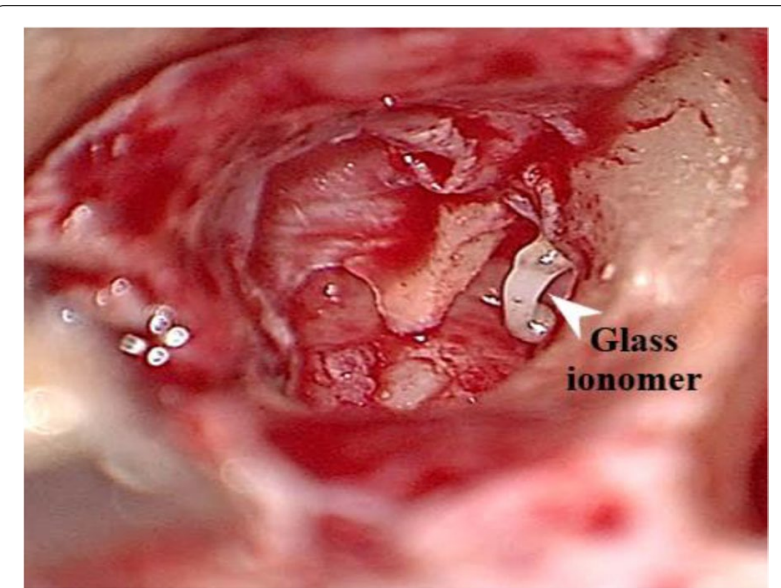

Fig. 3 Glass ionomer bridging the incus and stapes suprastructure 
was removed. Gelfoam was placed in the middle ear. The graft was placed by the underlay technique (Fig. 4).

Post-operative follow-up was done at 1-month, 3-month, 6-month, and 1-year intervals, and hearing was evaluated by doing pure tone audiometry with air conduction (AC) at $0.5,1,2,3,4,6$, and $8 \mathrm{kHz}$ and bone conduction at $0.5,1,2,3$, and $4 \mathrm{kHz}$. Pure tone average was calculated using $0.5,1,2$, and $3 \mathrm{kHz}$. Air-bone gap (ABG) was calculated and graft uptake and any other complications were noted.

\section{Results}

During the specified time period, 24 patients fulfilled the inclusion criteria. Majority of patients presented with a history of hearing loss (85\%), ear discharge (70\%), and ear pain (21\%). On otoscopic examination, 23 (95.8\%) patients had pars tensa perforation and $1(4.2 \%)$ patient had pars flaccida perforation. Of the 23 pars tensa perforations, 21 had subtotal perforations and 2 had large size perforation. On CT scan of the temporal bone, all 24 patients $(100 \%)$ had a suspicious long process of incus necrosis without evidence of any other bony erosion suggestive of cholesteatoma. On pure tone audiometry, the average AC threshold pre-operatively was $43.9 \mathrm{~dB}$. Student pair $t$-test was used to analyze the hearing improvement with a confidence interval set at $95 \%$ with $P<0.05$ being considered significant.

AC threshold changed from 43.9 to $23.13 \mathrm{~dB}$ at the end of 1 st month $(P \leq 0.0001)$ and to $15 \mathrm{~dB}$ at the end of 3rd month $(P<0.0001)$, showing significant hearing improvement.

Similarly, ABG improved from 23 to $15.5 \mathrm{~dB}$ at the end of 1 st month $(P=0.0004)$ and to $10.5 \mathrm{~dB}$ at the end of $3 \mathrm{rd}$ month $(P<0.0001)$ (Tables 1 and 2$)$. Twenty-two $(91.67 \%)$ patients had closure of $A B G<20 \mathrm{~dB}$, of which 13 (54.17\%) were male patients and 9 (37.5\%) were female patients.

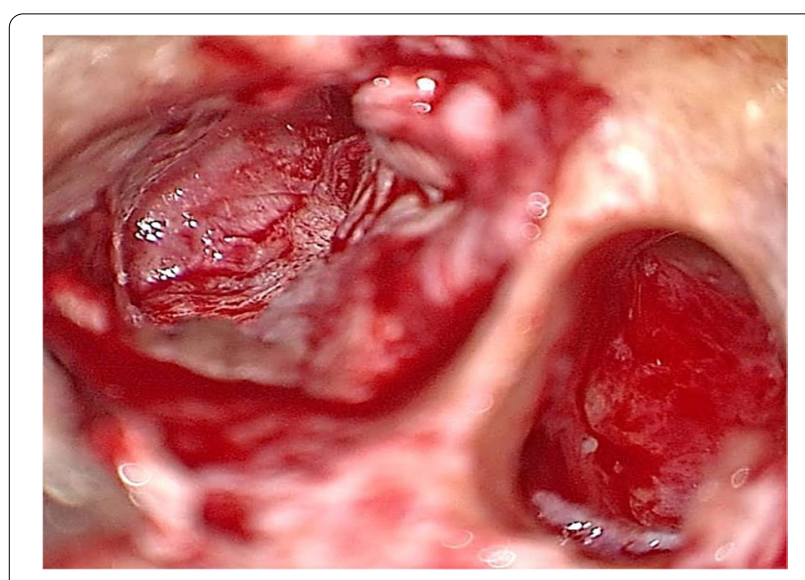

Fig. 4 Temporalis fascia graft placed by the underlay technique
Table 1 Post-operative air-bone gap at the end of 1st month

\begin{tabular}{ll}
\hline ABG value & $\begin{array}{l}\text { Number } \\
\text { of patients } \\
\text { (\%) }\end{array}$ \\
\hline$<10 \mathrm{~dB}$ & $7(29.2 \%)$ \\
$10-20 \mathrm{~dB}$ & $14(58.3 \%)$ \\
$21-30 \mathrm{~dB}$ & $2(8.3 \%)$ \\
$>30 \mathrm{~dB}$ & $1(4.2 \%)$ \\
\hline
\end{tabular}

Hearing was also evaluated at 6-month and 1-year intervals, which showed no deterioration in hearing (Table 3).

\section{Discussion}

The advantage of glass ionomer cement on the incudostapedial joint is mainly maintaining the normal ossicular assembly. It is recommended in cases where there is less than $1 / 3 \mathrm{rd}$ of the long process of incus erosion. It is not recommended in cases where there is severely eroded ossicular assembly or frank cholesteatoma. Glass ionomer is biocompatible, biostable, inert, moldable, cost-effective, and universally available. Our results have shown a definitive good post-operative hearing improvement during the 1-year follow-up. Incudo-stapedial joint reconstruction by glass ionomer can be more advantageous over partial ossicular replacement prosthesis (PORP). It gives more physiological continuity between the incus and stapes at the same time maintaining the incudo-malleolar axis. This helps attain the natural process of sound amplification. Incudo-stapedial joint reconstruction by glass ionomer has no extrusion which is an added advantage over PORP or TORP (total ossicular replacement prosthesis) [5-8]. Our study did not exhibit any adverse reaction to glass ionomer. A piece of gelfoam is kept under the incudo-stapedial joint during the procedure to prevent any spillage of the glass ionomer in the middle ear, stapes footplate, or suprastructure. For perfect alignment of the bonding, we used the slow bonding glass ionomer which gives the surgeon time to align the reconstructed joint ends [7] and clear accidental spillage if any.

Table 2 Post-operative air-bone gap at the end of 3rd month

\begin{tabular}{ll}
\hline ABG value & $\begin{array}{l}\text { Number } \\
\text { of patients } \\
\text { (\%) }\end{array}$ \\
\hline$<10 \mathrm{~dB}$ & $15(62.5 \%)$ \\
$10-20 \mathrm{~dB}$ & $7(29.16 \%)$ \\
$21-30 \mathrm{~dB}$ & $1(4.17 \%)$ \\
$>30 \mathrm{~dB}$ & $1(4.17 \%)$ \\
\hline
\end{tabular}


Table 3 Age, air conduction threshold, and air-bone gap of all the cases included in this study

\begin{tabular}{|c|c|c|c|c|c|c|c|}
\hline Serial number & Age & $\begin{array}{l}\text { Pre-op AC } \\
\text { threshold }\end{array}$ & 1-month AC threshold & 3-month AC threshold & ABG pre-op & ABG after 1 month & ABG after 3 months \\
\hline 1 & 35 & 45 & 23 & 14 & 22 & 9 & 9 \\
\hline 2 & 33 & 43 & 22 & 15 & 23 & 8 & 8 \\
\hline 3 & 15 & 44 & 21 & 16 & 24 & 9 & 7 \\
\hline 4 & 22 & 40 & 20 & 17 & 23 & 10 & 8 \\
\hline 5 & 26 & 46 & 24 & 15 & 25 & 20 & 10 \\
\hline 6 & 35 & 47 & 23 & 15 & 26 & 25 & 11 \\
\hline 7 & 34 & 45 & 23 & 14 & 23 & 32 & 31 \\
\hline 8 & 27 & 43 & 26 & 14 & 22 & 28 & 21 \\
\hline 9 & 44 & 38 & 27 & 14 & 21 & 14 & 9 \\
\hline 10 & 54 & 48 & 26 & 13 & 22 & 14 & 7 \\
\hline 11 & 52 & 45 & 25 & 18 & 21 & 15 & 8 \\
\hline 12 & 42 & 45 & 24 & 16 & 21 & 17 & 10 \\
\hline 13 & 45 & 46 & 23 & 16 & 23 & 8 & 7 \\
\hline 14 & 40 & 44 & 23 & 15 & 23 & 16 & 8 \\
\hline 15 & 30 & 43 & 22 & 15 & 25 & 8 & 7 \\
\hline 16 & 37 & 37 & 21 & 14 & 26 & 18 & 10 \\
\hline 17 & 55 & 46 & 21 & 15 & 23 & 9 & 7 \\
\hline 18 & 36 & 47 & 22 & 14 & 21 & 19 & 11 \\
\hline 19 & 35 & 46 & 22 & 16 & 23 & 19 & 13 \\
\hline 20 & 32 & 38 & 21 & 14 & 21 & 18 & 9 \\
\hline 21 & 30 & 45 & 26 & 16 & 23 & 17 & 15 \\
\hline 22 & 27 & 46 & 23 & 14 & 25 & 9 & 8 \\
\hline 23 & 34 & 47 & 26 & 16 & 23 & 19 & 9 \\
\hline 24 & 37 & 40 & 21 & 14 & 23 & 11 & 9 \\
\hline Total & 857 & 1054 & 555 & 360 & 552 & 372 & 252 \\
\hline Average & 35.71 & 43.9 & 23.13 & 15 & 23 & 15.5 & 10.5 \\
\hline Confidence interval & & & $95 \%$ & $95 \%$ & & $95 \%$ & $95 \%$ \\
\hline$P$ value & & & $<0.0001$ & $<0.0001$ & & 0.0004 & $<0.0001$ \\
\hline Significance & & & Extremely significant & Extremely significant & & Significant & Extremely significant \\
\hline
\end{tabular}

Abbreviations: $A C$ air conduction threshold, $A B G$ air-bone gap

This prospective observational study is conducted to see the efficiency of glass ionomer in incudo-stapedial joint restoration and the subsequent hearing improvement post-operatively.

Baglam et al. conducted a study on 136 patients of incudo-stapedial discontinuity reconstructed by glass ionomer of which $81.6 \%$ of patients attained postoperative $A B G$ of $<20 \mathrm{~dB}$ [9]. He also inferred that those having less than one-third of the long process of incus are ideal candidates for this technique of reconstruction and it did not interfere with graft uptake rate. In our study, we followed the same inclusion protocol.

In our study, the mean pure tone average pre-operative of the patients was 43.9 and the mean pure tone average post-operative at the end of 1st month and 3rd month was $23.13 \mathrm{~dB}$ and $15 \mathrm{~dB}$ respectively with $P$ value $<0.0001$ which showed significant hearing improvement. The post-operative ABG in our study was $<20 \mathrm{~dB}$ in $91.67 \%$ of patients of which $54.17 \%$ were male and $37.5 \%$ were female which correlates with the above study. These results were also similar to Brask et al. whose study on 44 patients showed post-operative $\mathrm{ABG}<20 \mathrm{~dB}$ in $83.3 \%$ of cases [10].

Babu and Seidman conducted a study on 80 patients with incudo-stapedial disarticulation using glass ionomer. The results showed improvement of ABG from a pre-operative average of 33 to $10 \mathrm{~dB}$ post-operatively [11]. In our results, there is an improvement of ABG from the pre-operative average of 23 to $10.5 \mathrm{~dB}$ post-operatively at the end of 3rd month $(P<0.0001)$, showing excellent hearing improvement. We did not 
experience any case of graft extrusion, foreign body reaction, infection, or any other complication.

\section{Conclusions}

Glass ionomer is a simple, easy, physiological, and costeffective method of ossicular reconstruction. This technique helps to maintain the natural ossicular assembly with good post-operative hearing improvement. It saves considerable operative time and money and hence more trials should be conducted using this method.

\section{Abbreviations}

ENT: Ear, nose, throat; OPD: Outpatient department; CT: Computed tomography; AC: Air conduction; ABG: Air-bone gap; PORP: Partial ossicular replacement prosthesis; TORP: Total ossicular replacement prosthesis.

\section{Acknowledgements}

Not applicable.

\section{Authors' contributions}

All authors contributed to the study conception and design. Material preparation, data collection and analysis were performed by MJ and BS. Both the authors contributed in writing the manuscript. All authors read and approved the final manuscript.

\section{Funding}

No funding was received to assist with the preparation of the manuscript.

\section{Availability of data and materials}

All data generated or analyzed during this study are included in this article.

\section{Declarations}

\section{Ethics approval and consent to participate}

This prospective observational study involving human participants was in accordance with the ethical standards of the institutional and national research committee and with the 1964 Helsinki Declaration and its later amendments or comparable ethical standards. The Bombay Hospital Ethics Committee approved this study with registration number ECR/296/Inst/ $\mathrm{MH} / 2013,15 / 01 / 2021$, Retrospectively registered. Informed written consent to participate in the study was provided by all participants or their parent or legal guardian in the case of children under 16.

\section{Consent for publication}

Written informed consent for the publication was provided by all participants or their parent or legal guardian in the case of children under 16.

\section{Competing interests}

The authors declare that they have no competing interests.

\section{Author details}

${ }^{1}$ Department of Otorhinolaryngology, Bombay Hospital and Medical Research Centre and Grant Medical College and J. J Group of Hospitals, Mumbai, India.
2 Juvekars Nursing Home, 17, 18 Shivsagar Society, Shivpuri, Off Sion Trombay Road, Chembur Naka, Chembur, Mumbai, Maharashtra 400071, India. ${ }^{3}$ Department of Otorhinolaryngology, Guwahati Neurological Research Centre, Kolkata, West Bengal, India.

Received: 5 August 2021 Accepted: 11 October 2021

Published online: 11 November 2021

\section{References}

1. Dr Jose Acuin. Chronic suppurative otitis media: burden of illness and management options; 2004. Chapter 1, p 21

2. Glasscock ME, Gulya AJ (2003) Surgery of the ear, 5th edn. BC Decker Inc., Hamilton, Ontario

3. El-Kashlan HK, Harker LA (2005) Tympanoplasty and ossiculoplasty. In: Cummings CW (ed) Otolaryngology head and neck surgery, 4th edn. Elsevier Mosby, United States of America, pp 3068-3074

4. Goycoolea MV (1989) Tympanoplasty. In: Goycoolea MV, Paparella MM, Nissen RL (eds) Atlas of otologic surgery, 1st edn. WB Saunders Company, United States of America, pp 218-246

5. Müller J, Geyer G, Helms J. Restoration of sound transmission in the middle ear by reconstruction of the ossicular chain in its physiologic position. Results of incus reconstruction with ionomer cement. Laryngorhinootologie 1994; 73(3):160-163. doi: https://doi.org/10.1055/s-2007-997102. PMID: 8172638

6. Geyer G. Implants in middle ear surgery. Eur Arch Otorhinolaryngol Suppl 1992; 1:185-221. PMID: 1521071

7. Feghali JG, Barrs DM, Beatty CW, Chen DA, Green JD Jr, Krueger WW et al (1998) Bone cement reconstruction of the ossicular chain: a preliminary report. Laryngoscope 108:829-836. https://doi.org/10.1097/00005537199806000-00010

8. Ramsden RT, Herdman RC, Lye RH. Ionomeric bone cement in neurootological surgery. J Laryngol Otol 1992; 106(11):949-953. doi: https://doi. org/10.1017/s0022215100121449. PMID: 1479268

9. Baglam T, Karatas E, Durucu C, Kilic A, Ozer E, Mumbuc S et al (2009) Incudostapedial rebridging ossiculoplasty with bone cement. Otolaryngol Head Neck Surg 141:243-246. https://doi.org/10.1016/j.otohns.2009.03. 025

10. Brask T (1999) Reconstruction of the ossicular chain in the middle ear with glass ionomer cement. Laryngoscope 109(4):573-576. 10201743. https://doi.org/10.1097/00005537-199904000-00010

11. Babu S, Seidman MD. Ossicular reconstruction using bone cement. Otol Neurotol 2004; 25(2):98-101. doi: https://doi.org/10.1097/00129492200403000-00003. PMID: 15021766

12. Niparko JK, Kemink JL, Graham MD, Kartush JM (1988) Bioactive glass ceramic in ossicular reconstruction: a preliminary report. Laryngoscope 98:822-825

\section{Publisher's Note}

Springer Nature remains neutral with regard to jurisdictional claims in published maps and institutional affiliations. 